\title{
Diagnostics of Transient States in Hydraulic Pump System with Short Time Fourier Transform
}

\author{
Krzysztof Przystupa1*, Bartłomiej Ambrożkiewicz', Grzegorz Litak¹ \\ 1 Department of Automation, Faculty of Mechanical Engineering, Lublin University of Technology, \\ Nadbystrzycka 36, 20-618 Lublin, Poland \\ * Correspondent author's e-mail: k.przystupa@pollub.pl
}

\begin{abstract}
In hydraulic pump system various states can occur caused by mechanical and physical phenomena. To detect them, the Short Time Fourier Transform (STFT) is applied. This paper will consider an application of STFT to monitor and evaluate hydraulic pump system operation in different states of operation. For measurements of pressure and flow changes in pump, hydraulic tester and Data AcQuisition (DAQ) card was used for evaluation of qualitative and quantitative changes in the system. Results of hydraulic pump's operation will be shown on Fast Fourier Transform (FFT) charts and STFT spectrograms plots.
\end{abstract}

Keywords: hydraulic pump system, FFT, STFT, diagnostics.

\section{INTRODUCTION}

Hydraulic pump systems are one of working systems commonly used in industry. It's selection for specific application mainly rely on its operational characteristics. Real shape of hydraulic pump characteristics depends on few phenomena (for instance flow resistance, recirculations), which occur during operating medium flow through it. To detect specific states in pumps, some methods of DSP (Digital Signal Processing) were already used [1-3].

In order to show new approach of diagnostics of transient states in hydraulic pump, the Short Time Fourier Transform was proposed. STFT has already been applied in health condition monitoring of working systems, for instance planetary gearbox [4] or detection of dynamic properties caused by shape errors [5].

Our problem considers operation of hydraulic pump system in different states of operation, both for normal operation and transient undesirable states [6]. Changes of pressure in pump and operating medium flow were imposed by regulating system, then the results were collected and put under DSP analysis. In our study we would like to point out, that STFT can be applied in diagnostics of dynamic changes in the test rig. Obtained spectrograms are used to estimate the frequency content of signals especially with low amplitude changing over time and this is an advantage over standard FFT method [7].

\section{SIGNAL PROCESSING}

Short Time Fourier Transform used to analysis of the hydraulic pump is one of the DSP methods dedicated for non-stationary signals [8]. The advantage of it is that we can obtain good timefrequency distribution for many constituent signals. In order to obtain information what frequencies are in specific time interval, small segment of the signal around it is considered. By changing time, a different signal spectrum is obtained. Entire spectrum shows how the spectrum changes in time and gives distribution of the time frequency. Short time segment compared to entire signal is the process of STFT. Time segment is calculated by multiplying the raw signal $s(\tau)$ by a window 
function $\mathrm{h}(\tau)$, concentrated around the time of interest $\tau$. Then we obtain weighted signal.

$$
s_{t}(\tau)=s(\tau) h(\tau-t)
$$

where: $s_{t}(\tau)$ weighted signal,

$$
\begin{aligned}
& s(\tau) \text { raw signal, } \\
& h(\tau-t) \text { window function. }
\end{aligned}
$$

The introduction process for the STFT in the discrete domain is performed by obtaining time window of the raw non-stationary signal S. Mainly Hamming of Gaussian window is used for convolving the signal. Then the DFT is calculated for each segment of the signal collected with applied window function to obtain STFT. The characteristic frequency of the signal is calculated from corresponding Fourier coefficient, which corresponds to the frequency.

$$
\omega_{i}=i \frac{2 \pi}{n \Delta t}
$$

where: $\omega_{i}$ Fourier coefficient,

$i$ number of element in STFT,

$n$ number of points in the window,

$\Delta t$ sampling rate of the signal.

One dimensional Fourier transform of the raw signal is considered as the window is slid along time axis, resulting in two-dimensional representation of the signal, what is actually STFT mathematically written as following [9].

$$
\begin{gathered}
\operatorname{STFT}\{s(t)\}(t, \omega)= \\
=\int_{-\infty}^{+\infty} s(t) h(t-\tau) e^{-j \omega t} d t
\end{gathered}
$$

\section{TEST RESULTS}

The test rig used for the experiment shown in Fig. 1 is used in pneumatics and hydraulics laboratory in Lublin University of Technology. Measurements of pressure and flow were performed in wide range of rotational speed of hydraulic pump $(0 \div 1050 \mathrm{rpm})$. Extortion of different states of hydraulic pump was regulated by changing position of hydraulic valve throttle.

The test equipment consisted of following elements indicated in Figure 1:

1. Hydraulic gear pump - Caproni 10A5X053G.

2. Hydraulic motor $-\mathrm{M}+\mathrm{S}$ MM8.

3. Flow meter - Stauff PPC-04/12-SFM-015

4. 3 Way Flow Control Proportional Valve Ponar WDUD10.

5. Hydraulic valve throttle - Ponar VRFB $90^{\circ} 3 / 8$ PN350.

6. Overflow valve.

7. Power system of cascade control system.

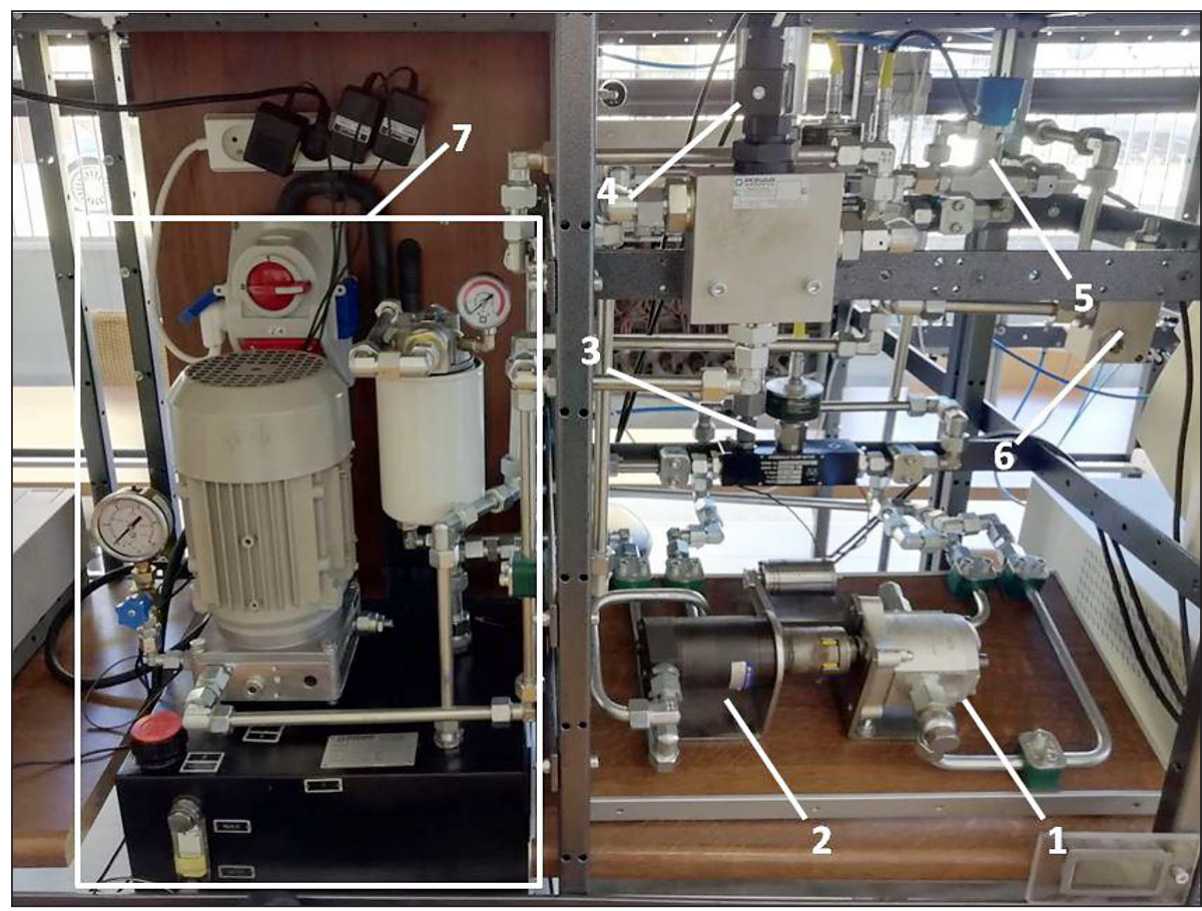

Fig.1. The testing rig system realizing cascade control system. 
The measurements of flow and pressure were conducted by using hydraulic tester STAUFF PPC-06/08-plus coupled with DAQ National Instruments 6341 . The sampling frequency was set to $10 \mathrm{kHz}$. To show system's possibility to adapt to different states, one long measurement showing several characteristic states was performed and its results later were taken for further signal analysis.

Obtained raw signal of pressure and flow is shown on Figure 2. Four states specific for hydraulic pump operation were found and indicated on it. They are the following:

1. Idle state - inifinitesimal flow, pressure value persisting on low level.

2. Impedance matching - flow and pressure on little bit higher level than in idle state.

3. Overload - pressure on maximum high level, flow level a little bit higher than in previous states.

4. Quasi-Cavitation - rapidly changing values of pressure and flow, signal of flow is high and unstable.

Above identified states of hydraulic pump system's operation can be described more precisely.

The first one is an idle state. It is characterized by the implementation of a rotational speed on the pump impeller at low torque. On the output of hydraulic pump, pressure is on the corresponding level defined by rotational speed and flow rate. As expected, this flow is very low (almost zero). Such state was indicated in Figure 2 as 1.
In the next state, impedance matching is reached by a coincidence of operation conditions of pump to the needs of working machine. Most often, the pressure level and pump flow rate are imposed by parameters of receiver. Namely, on the input of pump, the impedance matching state corresponds to a situation, where optimal shaft speed and torque occur. In Figure 2 this state is marked as 2.

On the other hand, exceeding the permissible working conditions leads to mechanical overload states of the pump. Such states are very dangerous and undesirable resulting in sealing damage. Then, the pressure increases significantly while fluid flow through pump decreases strongly. Depending on a mechanical operation, such a state corresponds to any rotational speed at high torque. It is indicated in Figure 2 as 3.

The last state marked in Figure 2 is marked as 4. It is a transient state, which can be determined as quasi-cavitation. This state is subjected to the influence of rapid acceleration of moving parts of the pump, they detach from the surface of the hydraulic medium as a result of its inertia. It is an undesirable phenomenon for the system's operation, because there are flow stream complex dynamics. Such stream shows nonhomogeneous areas of decreased density and even some areas with a lack of liquid, leading to vapor bubbles with a low pressure. Such a state in the laboratory conditions is difficult to obtain as it passes easily into a hydraulic impact. Hence here, a cavitation phenomenon was not distinguished from hydraulic impact. Therefore, we called the state 4 (Fig. 2)

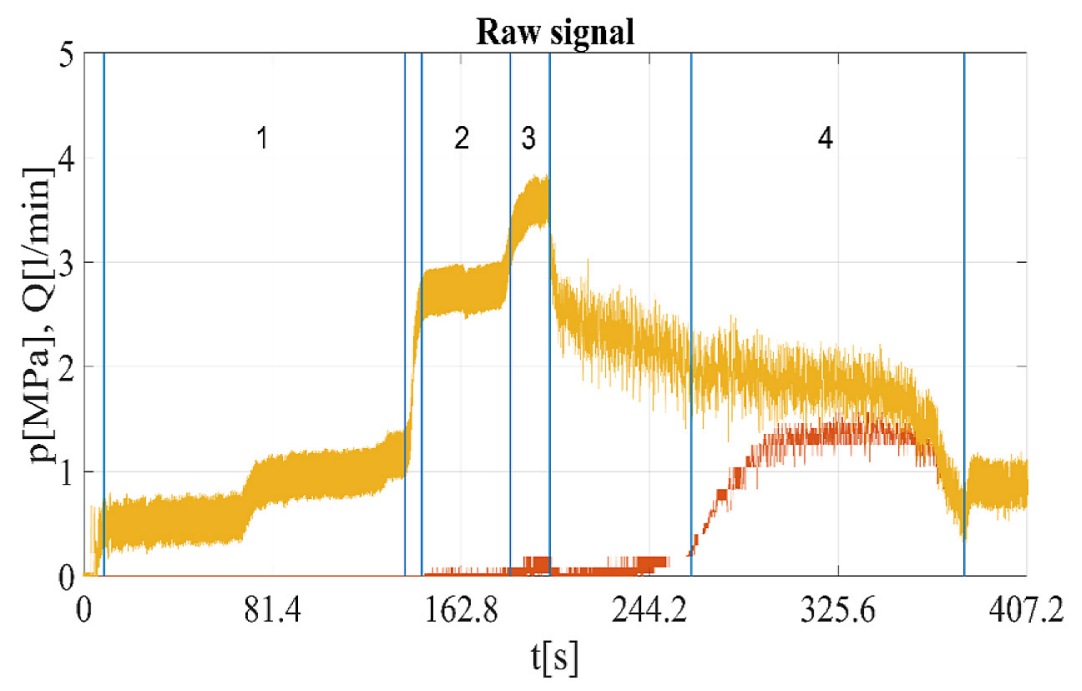

Fig. 2. Raw signal of hydraulic pump operation, yellow (pressure), red (flow rate). States identified in hydraulic pump are following: 1 - Idle state, 2 - Impedance matching, 3 - Overload, 4 - Quasi-Cavitation. 

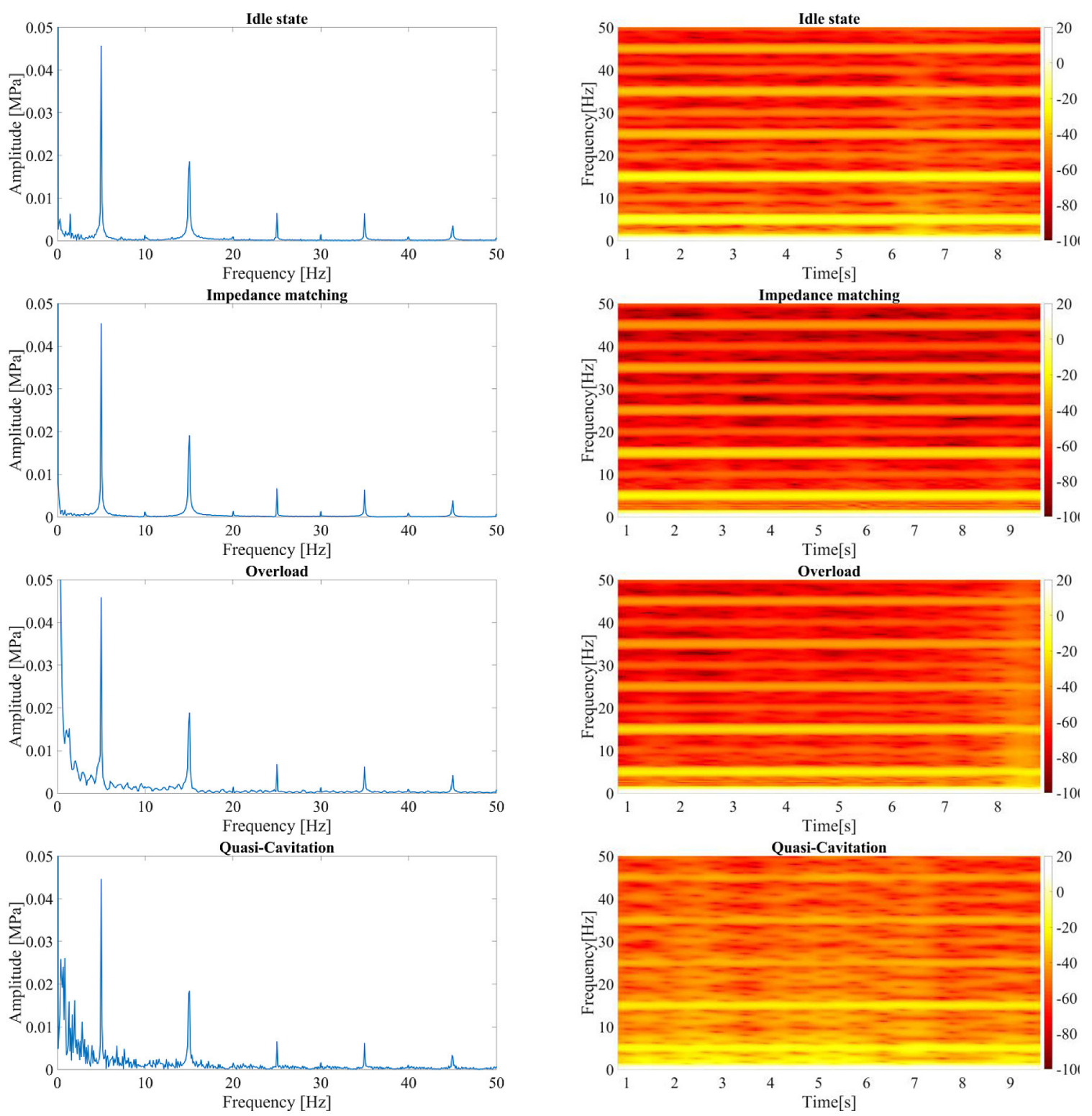

Fig. 3. Flow rate FFT (left) and STFT (right) plots for states identified in hydraulic pump operation (all the four cases as indicated in the figures)

as quasi-cavitation one. In the case of our experiment, this state was obtained by an extremely inaccurate selection of pump's operating parameters, its load and drive inputs. Obtained state is characterized by rapid changes in pressure accompanied by large changes in fluid flow [10-13].

\section{DISCUSSION}

States of hydraulic pump system operation defined in previous section were put under DSP analysis. For calculations 100000 results of pressure and flow were taken into account for each of states. At first, standard FFT algorithm was applied and next comparing it with STFT. To analyze our hydraulic pump dynamical responses, the Fourier transform was performed in a low range of the operational frequency, i.e. $0 \div 50 \mathrm{~Hz}$. Regarding results of pressure measurements, on FFT spectra it is hard to identify each of states. On charts clearly visible peaks come from operation of hydraulic pump starting from $5 \mathrm{~Hz}$ frequency and its higher harmonics. Many little peaks with low amplitude coming from pressure fluctuations for each state were found, what reflects obtained chart from the measurements (Fig. 2). Unfortunately, such little peaks are hardly visible on FFT spectrum and more accurate analysis is needed, so the STFT was applied. If it 

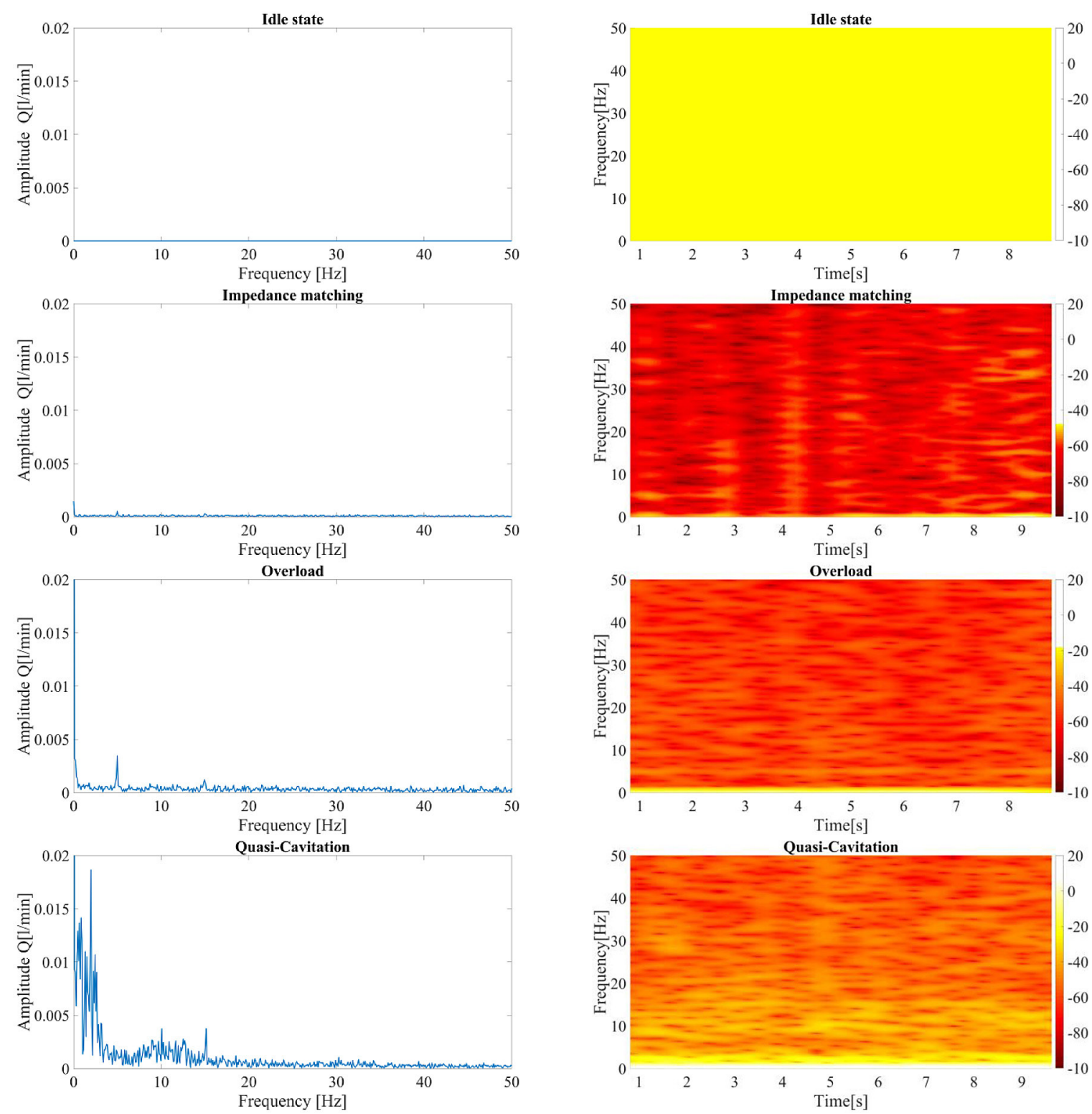

Fig. 4. Pressure FTT (left) and STFT (right) plots for considered states in hydraulic pump system (corresponding to the states presented in Fig. 3)

comes to results of analysis of flow in the pump, situation to identify rapid changes in flow is even harder. Like it was said in previous section in fluid flow through pump some transients physical phenomena can occur, so it is hard to obtain stable and proper results [14].

In our signal processing, we have focused on information coming from frequency domain, so applied length of window is fairly wide. We neglected information coming from time domain because the length of vector taken for calculations was the same and we did not take it into account. Using the Blackman window enables to reach advantages as higher accuracy, fairly good response in time and frequency domain and proper detection of peaks with fairly low magnitude [15]. Namely, STFT charts clearly show peaks obtained on FFT. A lot of little peaks with low amplitude and frequency occurring especially in idle and quasi-cavitation state are clearly visible also on STFT charts. The magnitude on spectrograms easily presents small peaks of FFT [16]. Comparing Figures 3 and 4 we conclude that a fluid flow rate is even harder to analyze with standard FFT, the differences between each state of operation are different in value of amplitude. With help of STFT, spectrum analysis is clearly shown. A fluid flow characterizes with many discontinuities and rapid changes, so in its case there are no specific peaks. 


\section{CONCLUSIONS}

Our comparative studies using FFT and STFT approaches show that the use of the second method has a practical application in the detection and identification of rapid pressure and flow changes in hydraulic pump systems. Flow and pressure disturbances are clearly visible for spectral analysis. STFT analysis is sufficient to identify the operating conditions of the pump in the hydraulic system, including diagnosing the conditions of the pump. When performing the assessment, it is important to select the function and width of the measurement window properly. The STFT method allows to capture and show fast and nonstationary signal changes on spectrograms. All four operating conditions of the hydraulic pump, defined by both pressure and flow changes, were identified and analyzed using a non-standard DSP method used in diagnostics.

\section{Acknowledgements}

The project was financed in the framework of the project Lublin University of Technology-Regional Excellence Initiative, funded by the Polish Ministry of Science and Higher Education (contract no. 030/RID/2018/19).

\section{REFERENCES}

1. Siano D., Frosina M. and Senatore A. Diagnostic Process by Using Vibrational Sensors for Monitoring Cavitation Phenomena in a Getoror Pump Used for Automotive Applications. In 72nd Conference of the Italian Thermal Machines Engineering Association, ATI2017, 6-8 September 2017, Lecce, Italy. Energy Procedia, 126, 2017, 1115-1122.

2. Jun S. and Kochan O. Common mode noise rejection in measuring channels. Instruments and Experimental Techniques, 58(1), 2015, 86-89.

3. Liu Y., Yao E. and Xu H. Fault diagnosis of hydraulic pump based on particle filtering and autoregressive spectrum. Chinese Journal of Scientific Instrument, 33(3), 2012, 561-567.

4. Bartelmus W. and Zimroz R. Vibration consition monitoring of planetary gearbox under varying external load. Mechanical Systems and Signal
Processing, 23, 2009, 246-257.

5. Nandi S., Ilamparithi T.C., Lee S.B. and Hyun D. Detection of Eccentricity Faults in Induction Machines Based on Nameplate Parameters. IEEE Transactions on Industrial Electronics, 58(5), 2011, 1673-1683.

6. Przystupa K. An attempt to use FMEA method for an approximate reliability assessement of machinery. EDP Sciences, 15, 2017.

7. Gutten M., Korenciak D., Kucera, M., Janura, R., Glowacz, A. and Kantoch, E. Frequency and time fault diagnosis methods of power transformers. Measurement Science Review, 18(4), 2018, 162-167.

8. Griffin D.W. and Lim J.S.. IEEE Transactions on Acoustics, Speech and Signal Processing, 32(2), 1984.

9. Yeromenko, V. and Kochan, O. The conditional least squares method for thermocouples error modeling. IEEE 7th International Conference on Intelligent Data Acquisition and Advanced Computing Systems (IDAACS), 1, 2013 157-162.

10. Del Campo D., Castilla R., Rausch G.A., Gamez Montero P.J. and Codina E. Numerical analysis of external gear pumps including cavitation. Journal of Fluids Engineering, 134(8), 2012.

11. Przystupa K. Reliability assessment method of device under incomplete observation of failure. In 2018 18th International Conference on Mechatronics-Mechatronika (ME) IEEE, 2017, 1-6.

12. Przystupa K., Pohrebennyk V., Kochan O. and Jun $\mathrm{S}$. Methods and Means of Measuring the Vortex Component of the Flow Velocity. 12th International Conference on Measurement, 2019, 236-239.

13. Pogrebennik, V.D. Investigation of systematic errors in measurements of the rotational component of a flow velocity. Measurement techniques, 40(9), 1997, 904-911.

14. Wang J., Kochan, O., Przystupa, K. and Su, J. Information-measuring System to Study the Thermocouple with Controlled Temperature Field. Measurement Science Review, 19(4), 2019, 161-169.

15. Podder P., Khan T.Z., Khan M.H. and Rahman M.M. Comparative Performance Analysis of Hamming, Hanning and Blackman Window. International Journal of Computer Applications, 96(18), 2014.

16. Glowacz, A. Recognition of acoustic signals of loaded synchronous motor using FFT, MSAF-5 and LSVM. Archives of Acoustics, 40(2), 1997, 197-203. 